\title{
Analysis about the Influence of the Damping Structure on the Mechanical Property of Roller
}

\author{
Qi Yingchun ${ }^{1, a}$, Jin Jingfu ${ }^{1, b}$, Cong Qian ${ }^{2, c}$ \\ ${ }^{1}$ School of Biological and Agricultural Engineering, Jilin University, Jilin Changchun, 130022, China \\ ${ }^{2}$ Key Laboratory of Engineering Bionics Minisity of Education, Jilin University, Jilin Changchun, \\ 130022, China \\ aqiyc@jlu.edu.cn, bjin Jingfu@jlu.edu.cn, Congqian@jlu.edu.cn
}

Keywords: bionic coupling; damping; wear; numerical analysis; roller

\begin{abstract}
The falling of wear layer on the surface of roller is a main form of roller failure. Based on the typical structure of normal roller and bionic coupling theory of structure and material, a coupling roller with strips on the surface of damping layer is designed. To study the mechanical property of the coupling roller, several numerical computations are carried out depending on the boundary condition and force loader of the working roller. The results prove that this kind of coupling roller has a better performance in reducing the possibility of crack generation by decreasing the difference of stress and strain gradient in the contact area of damping layer and wear layer and the size of the strips can also affect the ability of relatively soft damping layer material to reduce the stress impact.
\end{abstract}

\section{Introduction}

As the key working part of many grinding equipments, the roller is produced by orderly welding transition layer, damping layer, wear layer and surface pattern layer on the substrate [1]. But after working under the high pressure cyclic loading for a long time, abrasion and falling of wear layer are often happened. Usually, the abrasion hardness of wear layer is higher than that of damping layer, but the toughness of damping layer is better than that of wear layer because of the different function of these layers on the roller. And the differences of these material properties are also the reason of the falling off of wearing layer. The transmit of higher stress within different materials produces big change of stress and strain on the binding site of damping layer and wear layer. Some crack will be generated on this place and with the increase of working hours these cracks will eventually lead to falling of wear layer. The more cracks, the greater of the chance of wear layer off. A systematic and detailed study has been done by some professors in Jilin University and other institutes through analyzing the relationship between the combing of soft and hard material or structure and its influence to their mechanical properties [2-5]. Based on the structural bionics and material bionics, a coupling roller with non smooth damping layer and wear layer was designed depending the damping property of tenacity or relatively soft material. By computing the stress transmits nature of this roller, it is believed that buffer performance of soft material can be better used to reduce the stress gradient and strain gradient and decrease the rate of crack generation through changing the contacting form of these two layers. And these changes are very useful in lowering the rate of wear layer falling off. 


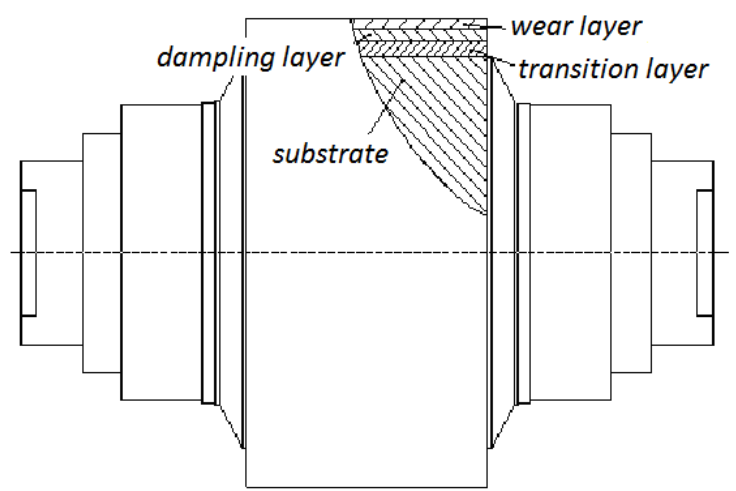

Fig1. Hard surfacing intake roller

\section{Structure Design of Damping Layer}

There are many samples for using the combining of soft and hard things to improve the wear property and shock resistance in the nature environment. For example, lizard living in desert, in order to survive in the wicked sand blown by the wind, there are multilayered structure exiting on lizard body surface [5, 6]. These diamond scale attached to the surface of the lizard skin are linked together by the skin and form a special body surface that represents a kind of system coupling of flexible absorbing and rigid reinforcing. This kind of coupling system of material, shape and structure can resist abrasive wear and erosive wear. A research group leading by Academician Ren Lu-quan designed and produced some element bionic sample pieces and coupling bionic sample pieces. They proved that a $20 \%$ improvement of erosion wear resistance can be gained for groove shape bionic sample and a 10\% improvement of erosion wear resistance can be gained for shell coupling bionic sample. And the maximum improvement erosion wear resistance about $39 \%$ can be gain for structure and material coupling bionic sample [6].

A new coupling bionic drill bit impregnated with a diamond used at gas well was designed by a research group leading by Professor Sun You-hong. Compared with normal drill bit, this coupling bionic drill bit has a faster rate of penetration about $42.7 \%$ and its working longevity is increasesd by 73.8\%.[7]

Shells get the attention of many researchers because of the perfect mechanical property of their nacreous layer. Ultra-thin layers of calcium carbide that are connected together by less than one-billionth of a meter of organic protein matrix can be found through the microscopic observation of shell transverse cross section. This structure allows the strength of the shell and the ceramic material quite and not prone to broken. The soft organic protein matrix exiting within the frame of hard calcium carbide plays obvious buffer action and improves their touch abradability. Different sample of bionic coupling laser were produced by Zhou Hong through the coupling of different elements of material, shape and structure based on shells biological prototype. The experiment results prove that under the guidance of bionic coupling theory, the abrasion resistance of the prepared specimen bionic coupling laser was greatly enhanced [8].

According to the research results about structure, shape and material coupling, a non smooth structure at the contacting area between damping and wear layer is designed based on the working characteristics and functions. Uniform strip structures are added on the surface of damping layer and then wear layer is welded on this surface. This process will make the contact area of both layer larger and relatively soft damping material will extend to the hard wear layer. When higher cycle stress transmits to this area, amount energy will be absorbed in different place and time and this will decrease the interaction force of damping layer and wear layer and then reduce the rate of crack generation probability. (As shown in Fig2). 


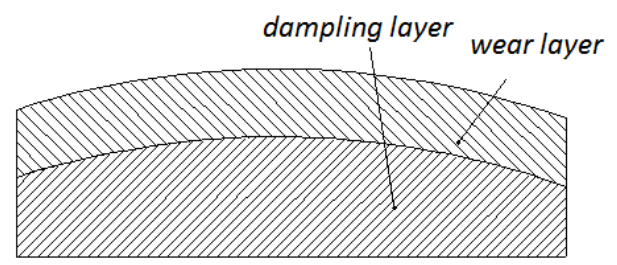

a. normal dampling and wear layer structure of roller

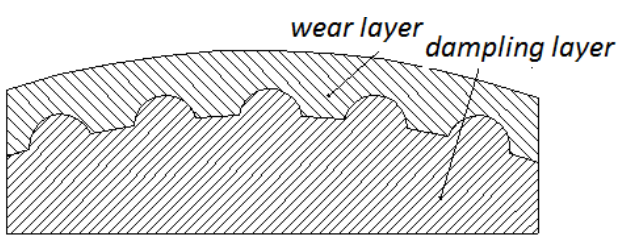

b. coupling non-smooth roller

Fig2. The structure of coupling roller

\section{Mechanics Property Analysis}

In order to study the influence of non-smooth structure on the internal stress delivery within damping layer and wear layer, a numerical analysis on the force of the coupling roller during work state is carried out.

\section{Analytical Model}

During working, both radial direction extrusion force $\mathrm{N}$ and tangential friction force $\mathrm{f}$ are acted on the roller surface. There is an intersection angle a between resultant force $F$ and extrusion force N(shown in Fig3). The size of the angle a depends on the friction coefficient between roller and pulverizing material. Normally, scope of this angle is $-3^{\circ}-7^{\circ}$.

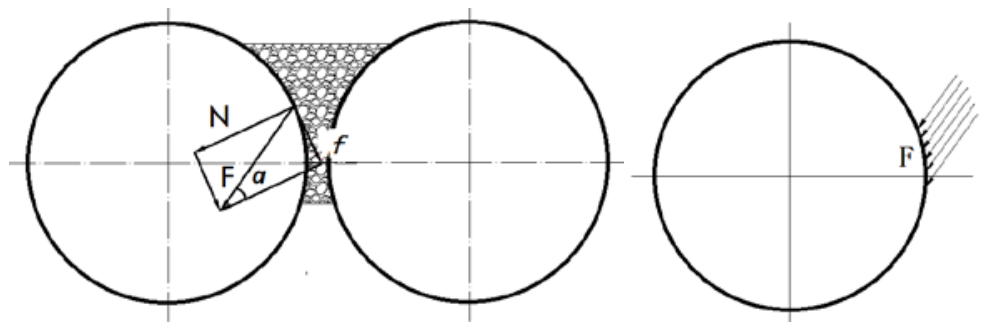

Fig3. Force state of the roller

Table1 the structure size and material properties of the roller

\begin{tabular}{|c|c|c|c|c|c|}
\hline \multirow[t]{2}{*}{ the roller size } & \multirow{2}{*}{$\begin{array}{c}\text { Working } \\
\text { paramete } \\
r\end{array}$} & \multicolumn{4}{|c|}{ material properties } \\
\hline & & & Young`s modulus(MPa) & Poisson`s Ratio & Density $\mathrm{kg} / \mathrm{m}^{3}$ \\
\hline \multirow{2}{*}{$\begin{array}{l}\text { diamerer:1400mm; } \\
\text { length: } 1200 \mathrm{~mm} \text {; } \\
\text { thickness of } \\
\text { wear-layer:15mm }\end{array}$} & $\begin{array}{c}\mathrm{F}=100 \\
\mathrm{MPa}\end{array}$ & $\begin{array}{c}\text { Damping } \\
\text { layer }\end{array}$ & 68000 & 0.3 & 2.7 \\
\hline & & Wear layer & 208000 & 0.34 & 7.86 \\
\hline
\end{tabular}
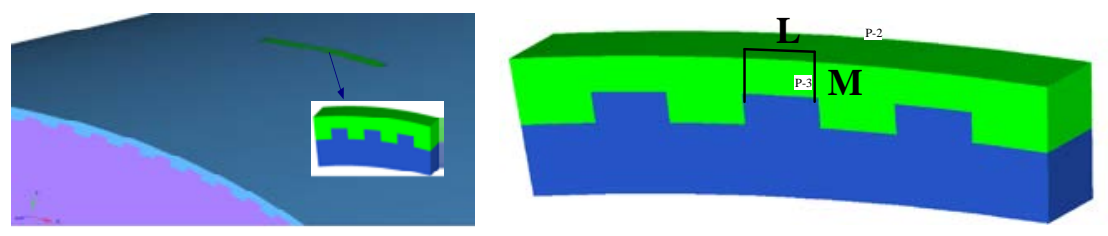

Fig4. Analytical model

L- The width of raised structure; $\mathrm{M}$ - the height of raised structure

For researching the affect of the size of non smooth raised structure to the transmitting of internal stress, the height and width of the raised structure are designed to change regularly. The heights are $2 \mathrm{~mm} 、 4 \mathrm{~mm} 、 6 \mathrm{~mm} 、 8 \mathrm{~mm} 、 9 \mathrm{~mm}$ and the widths are $6 \mathrm{~mm} 、 18 \mathrm{~mm} 、 25 \mathrm{~mm} 、 30 \mathrm{~mm} 、 35 \mathrm{~mm}$. To show the detailed information of the analysis results, a cube of $30 \mathrm{~mm}$ high, $15 \mathrm{~mm}$ wide and 120 long is cut out in the axial center section of the roller. 


\section{Analysis of the Result}

The distribution trend of the stress on the surface and body of roller is similar with the force distribution loaded on the roller. As the direction of the force loading is out of the vertical of the roller surface, the direction of the stress distribution has also slope with the surface normal direction. Show in Fig. 4.and Table 2. The force loaded on the surface of the roller passes through the wear layer and then goes into the damping layer and transition layer. Extended oblique stress makes the material of the roller undertake both tangential force and normal force that also have a tilt with the direction of the surface normal direction or the radial direction of the roller and this situation can increase the possibility of crack generating within the roller especially in the binding site of different material because of their different property. If there are amounts of crack, the probability of roller surface falling will enlarge. In order to decrease the possibility of crack generation, it is hoped that the inner stress can go toward radial direction of the roller.

The stress cloud and strain cloud of normal roller and coupling roller with different strip heights are given in Table2. All the computations are carried out under same boundary conditions and loaders. Because of the different material attribute, the average stress within wear layer is obvious larger than that within damping layer as the elasticity modulus of the wear layer is bigger. But the average strain within damping layer is greater and this is more beneficial to absorb the impact force passed from the wear layer.

The distribution gradient of inner stress and strain within wear layer and damping layer of normal roller is very clear and order. With the increase of the strip height on the damping layer, the inner stress within the damping layer also increases and tends to uniform. Correspondingly, the average inner strain and its gradient within the damping layer decrease and also tend to more uniform. The difference of the average stresses between the two layers become smaller. The reducing of stress and strain gradient is favorable to decrease the interaction between the material of two layers and the generation of cracks. Although the maximum stresses in both layers of coupling roller are bigger than that of normal roller, these values are within the limitation of their materials. So it is useful and reasonable to add strips on the damping layer to decrease the possibility of the crack generation and the falling of wear layer. It can be seen from Fig.5.

Table 2 stress cloud and strain cloud of roller with different damping strips height

\begin{tabular}{|c|c|c|}
\hline height of strip (mm) & stress cloud chart & strain cloud chart \\
\hline 0 & 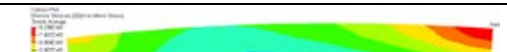 & 要 \\
\hline 2 & 策 & $\sqrt{v_{1}}$ \\
\hline 4 & & \\
\hline 6 & & \\
\hline 8 & & \\
\hline 9 & 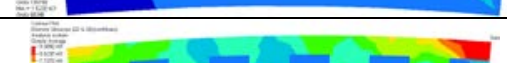 & \\
\hline
\end{tabular}

The stress cloud and strain cloud of normal roller and coupling roller with different strip widths are given in Table3 and also all the computations are carried out under same boundary conditions and loaders. It can be seen from the maximum stress and strain values and their clouds that the affect of 
the width of the strips to the inner stress and strain within two layers increase with the growth of the width of the strips. Following the enlarging of the strip width, the inner stress and strain within damping layer strengthen and their gradient reduces and the stress and strain gradients within the contact area of both layers reduce too. This means that the intensity or strength of the contact area becomes higher and its anti-load capacity is better. It can be seen from Fig.6

Table 3 stress cloud and strain cloud of roller with different damping strip width

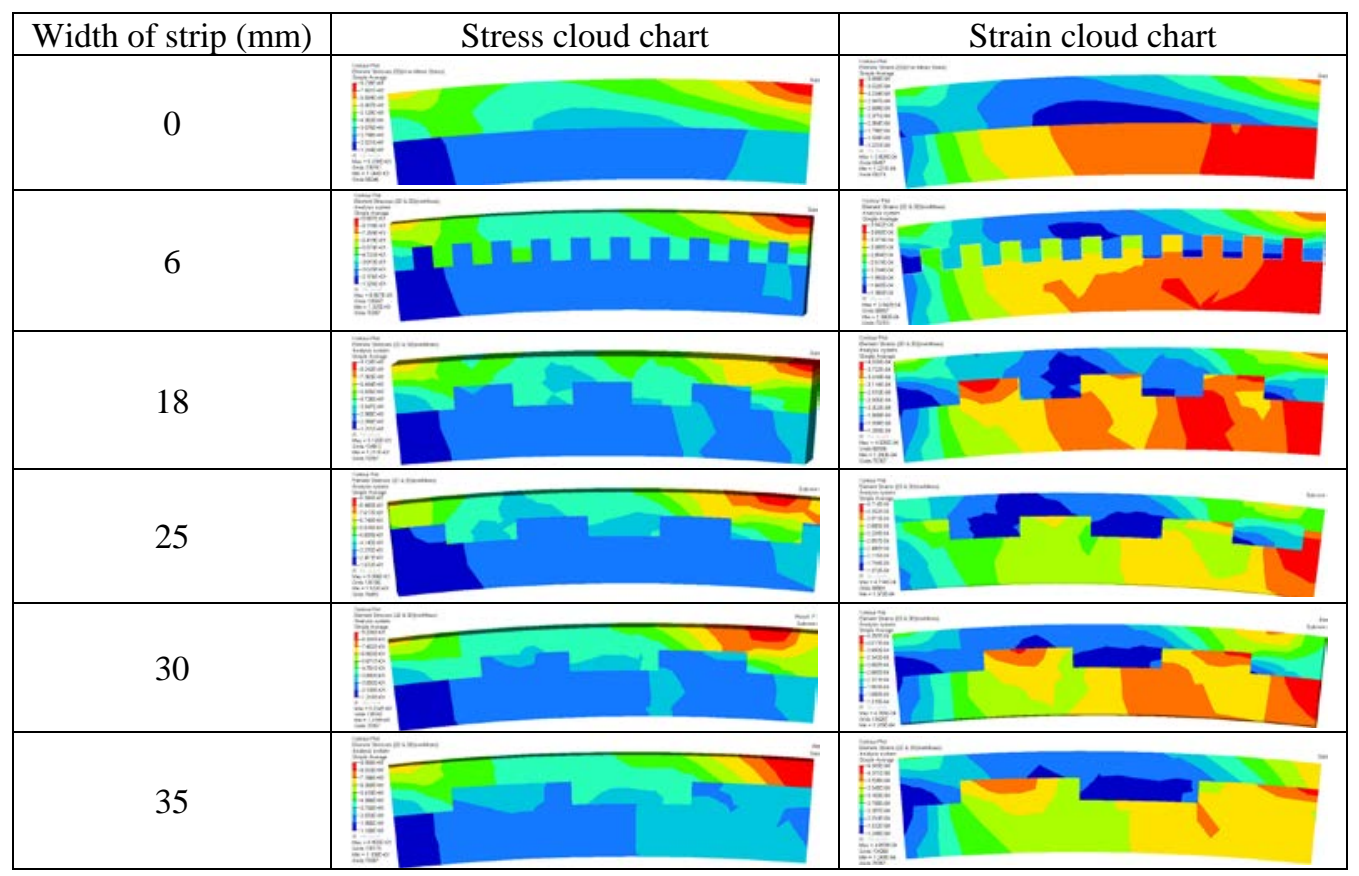

It is believed that soft materials have better ability to absorb impact energy because of their easier deformation and this ability makes them more useful in buffer or damping situation. This theory is also feasible in heavy load conditions such as roller. It can be seen from the computations that adding strip on the surface of the damping layer has basically no effect on the working performance of wear layer who has more contributes to smash the pulverizing material. But these strips elevate the ability of damping layer absorbing energy by increasing the stress and strain of damping layer and in the same time decrease the difference of strain gradient of both layers. All these effects are beneficial to reduce the interaction of dissimilar materials and consequently reduce the chance of crack initiation. So academically, it is possible to increase the ability to resist fatigue load of roller by adding strips on the damping layer or changing the structure of the damping layer.

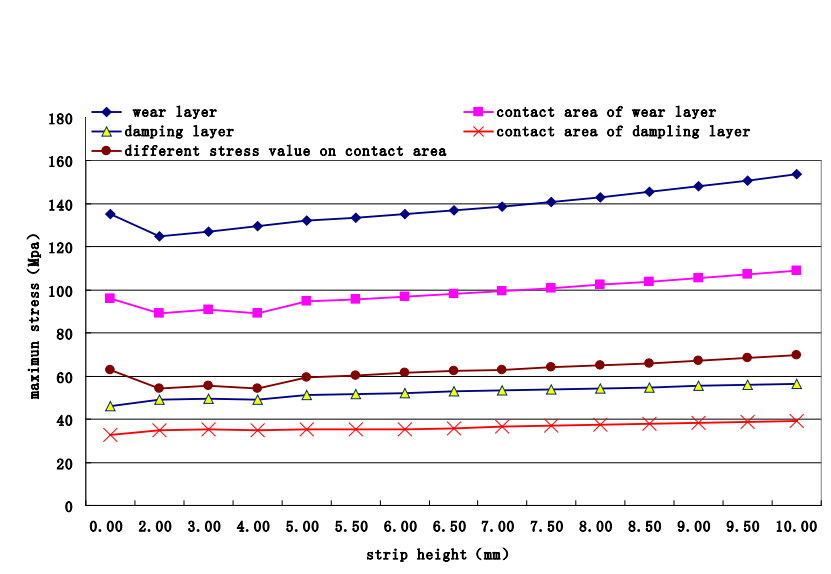

Fig5. maximum stress curves for rollers with different strip height

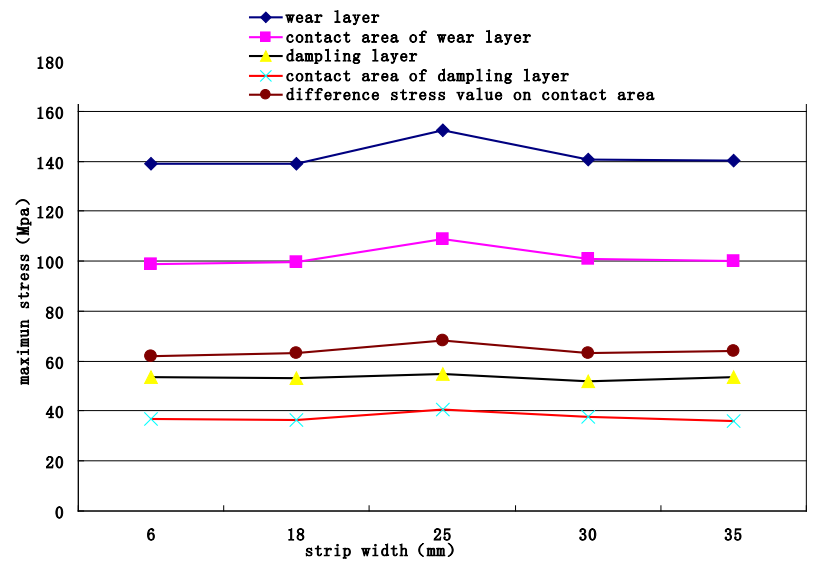

Fig6. maximum stress curves for rollers with different strip width 


\section{Conclusion}

The falling of the wear layer is a main factor that affects roller's working life. According to the structure of the roller, several coupling rollers are design based on the research of shape, structure and material coupling by adding strips on the surface of damping layer to change the contact situation of damping layer and wear layer. The stress and strain states of the coupling rollers with different strip sizes are analyzed and the results prove that this design is feasible in theory and the size of the strip has great effect to the stress distribution and its gradient. The coupling roller has a less possibility to initiate cracks that come from the higher force from the extrusion of pulverizing material than that of normal roller.

And also there are some other things that need to be carried out for a more detailed study of the coupling roller such as the manufacture of the coupling roller and the influence of the strips of the damping on the welding of different material. These will be the next steps that our research group will do.

This work provides a theoretical basis to study the possibility of reducing the possibility of the falling of wear layer on the roller by changing the structure of the damping layer but without affecting the performance of roller pulverizing material.

\section{Acknowledgement}

This study was supported by the National Natural Science Foundations of China (Grant No. 51205157).

\section{References}

[1] Xiaohong Jiang, Ping Ye, Zongkai Li. Analysis of Grinding Roller Stress Distribution of Vertical Roller Mill[J] .Advanced Materials Research 2014-06-30,Vol. 936, pp 1862-1867

[2] Brian Dean, Bharat Bhushan .The effect of riblets in rectangular duct flow[J] Applied Surface Science ,258 (2012) 3936 - 3947

[3] Baumgartner W, Saxe F, Weth A, Hajas D,Sigumonrong D, Emmerlich J,Singheiser M, B W, Schneider J M. The Sandfish's Skin: Morphology,Chemistry and Reconstruction. Journal of Bionic Engineering . 2007

[4] Song Qi-fei, Liu Yong-bing,Ahou Hong,Sun Na,Ren Lu-quan. Friction and wear behavior of bionic coupling brake drum processed by laser[J].Journal of Jilin University(Engineering and Technology Edition). 2007.9,Vol37(5):1070-1073

[5] L Q Ren, Z W Han. The multi-element coupling analysis of typical plant leavesbased on analysis hierarchy process (AHP) [C]. Pro-ceedings of the 2nd International Conference of Bion-ic Engineering, 2008.

[6] Gao Feng,Huang He,Ren Lu-quan,Erosive wear resistance of Laudakin stoliczkana's ternary coupling and bionic experiments[J]. Journal of Jilin University Engineering and Technology Edition

[7] Y Zhang, C H Zhou, L Q Ren. Biology couplingcharacteristics on soil-engaging components of molecrickets [J]. Journal of Bionic Engineering, 2008, 5:164-171.

[8] Xu Liang, Sun You-hong. Gao Ke. Efficient Rock Fragmentation Mechanism Analysis of Impregnated Diamond Bionics Bit[J] Journal of Jilin University(Earth Science Editon). 2008.11, Vol.38 (6):1015-1019

EMAIL: QIYC@JLU.EDU.CN

PHONE: 13500807062 